\title{
Metabolism under the spotlight in senescence
}

Corine Bertolotto

bertolot@unice.fr

Coverage on: Kaplon J, Zheng L, Meiss K, Chaneton B, Selivanov VA, Mackay G, van der burg SH, Verdegaal EME, Cascante M, Shlomi T, Gottlieb E and Peeper DS. (2013), A key role for mitochondrial gatekeeper pyruvate dehydrogenase in oncogene-induced senescence, Nature, 498:109-112. doi: 10.1038.

Senescence is a failsafe mechanism that represents a potent barrier against oncogenic transformation. Cells may undergo replicative senescence caused critical telomere shortening which occurs at each round of cell division but they can also be blocked in premature senescence by oxidative stress or oncogenic stimuli. Human nevi are one of the best examples of senescence in vivo. Nevus cells frequently express oncogenic NRAS ${ }^{\mathrm{Q} 61 \mathrm{~K}}$ or $\mathrm{BRAF}^{\mathrm{V} 600 \mathrm{E}}$ and are thought to be arrested in growth because of Oncogene-Induced-Senescence (OIS). Nevi typically remain growth arrested for decades, thereby indicating that senescence provides an efficient suppression of cell proliferation and tumorigenesis.

Senescence bypass is required for malignant transformation, and, accordingly,

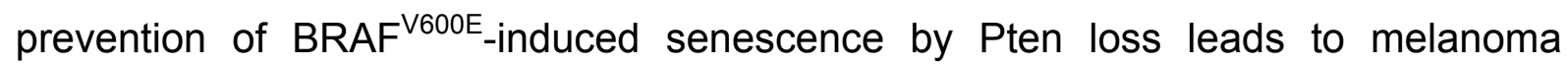
progression (Vredeveld et al 2012). However, the mechanisms that contribute to senescence bypass are not fully understood-unraveling those mechanisms can be important for a better understanding of melanoma biology, and also to unveil potential therapeutic targets.

Replicative and premature senescence share similar features. The cells increase in size, flatness, granularity and exhibit a senescence-associated ßgalactosidase (SA-ß-gal) activity. They are also characterized by chromatin modification, changes in gene expression and a growth arrest even in a strong mitogenic environment. However, despite the block in proliferation, senescent cells are viable and metabolically active. Little information was currently available on the metabolic changes that are linked and therefore may contribute to OIS.

Kaplon et al. show that human diploid fibroblasts (HDF) undergoing

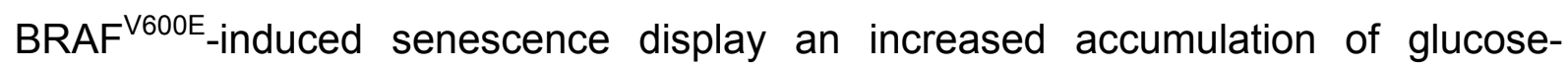


derived TCA cycle metabolites and an enhanced oxygen consumption rate (OCR), hallmarks of oxidative phosphorylation. The authors demonstrate that pyruvate dehydrogenase (PDH), which links glycolysis to the oxidative metabolism, has a causal role in the metabolic change and in establishing the OIS. Inhibition of Pyruvate dehydrogenase phosphatase 2 (PDP2) or forced expression of pyruvate dehydrogenase kinase 1 (PDK1), two enzymes that positively or negatively regulate PDH activity respectively, impair the mitochondrial oxidative phosphorylation phenotype and promote OIS escape. As expected, conversely PDK1 depletion triggers cellular senescence that is associated with an enhanced PDH activity and OCR. The authors next investigated the effect of PDK1 in melanocytes. They showed that xenografts of $p 53$ depleted;BRaf ${ }^{\mathrm{V} 600 \mathrm{E}}$ mouse melanocytes with forced expression of PDK1 formed tumor, thereby demonstrating that PDK1 can induce tumorigenicity. Inversely, xenografts of melanoma cells with PDK1 suppression into nude mice displayed limited growth compared to the control cells. Finally, experiments using cultured cells indicated that PDK1 knockdown enhanced the effect of BRAF inhibitor in both sensitive and resistant $\mathrm{BRAF}^{\mathrm{V} 600 \mathrm{E}}$-mutated human melanoma cells.

In essence, Kaplon et al reveal that OIS is associated with a metabolic rewiring, linked specifically to the PDK1-PDP2-PDH axis, which is instrumental in promoting the program of senescence in HDF (Figure 1). Similarly, a high rate of basal oxygen consumption in fibroblasts that have undergone Ras-induced senescence has been previously reported.

There are still several unanswered questions such as whether this metabolic rewiring also occurs and is instrumental in melanocytes experiencing OIS and what is the metabolic status of cells derived from human nevus versus melanocytes. How oncogenes control the expression/activity of the metabolic enzymes in order to mediate this metabolic rewiring characterized by elevated PDH activity and OCR needs to be determined.

Moreover, the role of PDK1 in melanoma cells remains to be fully elucidated. On one hand, Kaplon et al. showed that PDK1 depletion, which is expected to increase the level of oxidative phosphorylation, triggers melanoma cell death. On the other hand, melanoma cells are characterized by an elevated level of oxidative phosphorylation (Barbi de Moura et al 2012). Induction of cell death upon PDK1 depletion suggests that PDK1 could exert its effect in an oxidative phosphorylationindependent manner. Finally, it will be important to assess the effect of PDK1 
depletion in BRAF wild-type melanoma cells, which represent half of all the melanoma cases, and for which there are no effective cures.

Interestingly, although most cancer cells have conceivably bypassed OIS, several reports indicate that they are still able to enter senescence in response to chemotherapy drugs, a process called therapy-induced senescence (TIS) (Ohanna et al 2011, Schmitt et al 2002). Tumors capable of drug-induced senescence have a much better prognosis following chemotherapy (Schmitt et al 2002). However senescent cells are endowed with potentially harmful properties that likely contribute to long-term tumor progression. Recently, the group of C. Schmitt has also shown that TIS lymphomas, like OIS fibroblasts, exhibit metabolic reprogramming, reflecting increased glucose utilization and energy production. They elegantly demonstrate how targeting this hypermetabolism, which is linked to a massive proteotoxic stress occurring during senescence, induces tumor regression (Dorr et al 2013). Notably, TIS in melanoma cell lines also triggers the hypermetabolic phenotype, and share the vulnerability towards agents that target glucose utilization or other components of the hypermetabolic response.

In conclusion, recent reports demonstrate that senescence is associated with a metabolic reprogramming that might be therapeutically exploited to eradicate tumor cells. Both the groups of D. Peeper and C. Schmitt provide specific examples how conceptually novel therapeutic strategies that take advantage of senescenceassociated metabolic reprogramming might improve melanoma treatment.

\section{References}

Barbi de Moura M, Vincent G, Fayewicz SL, Bateman NW, Hood BL, Sun M et al (2012). Mitochondrial respiration--an important therapeutic target in melanoma. PloS one 7: e40690.

Dorr JR, Yu Y, Milanovic M, Beuster G, Zasada C, Dabritz JH et al (2013). Synthetic lethal metabolic targeting of cellular senescence in cancer therapy. Nature.

Ohanna M, Giuliano S, Bonet C, Imbert V, Hofman V, Zangari J et al (2011). Senescent cells develop a PARP-1 and nuclear factor-\{kappa\}B-associated secretome (PNAS). Genes Dev 25: 1245-1261.

Schmitt CA, Fridman JS, Yang M, Lee S, Baranov E, Hoffman RM et al (2002). A senescence program controlled by p53 and p16INK4a contributes to the outcome of cancer therapy. Cell 109: 335-346. 
Vredeveld LC, Possik PA, Smit MA, Meissl K, Michaloglou C, Horlings HM et al (2012). Abrogation of BRAFV600E-induced senescence by PI3K pathway activation contributes to melanomagenesis. Genes Dev 26: 1055-1069. 


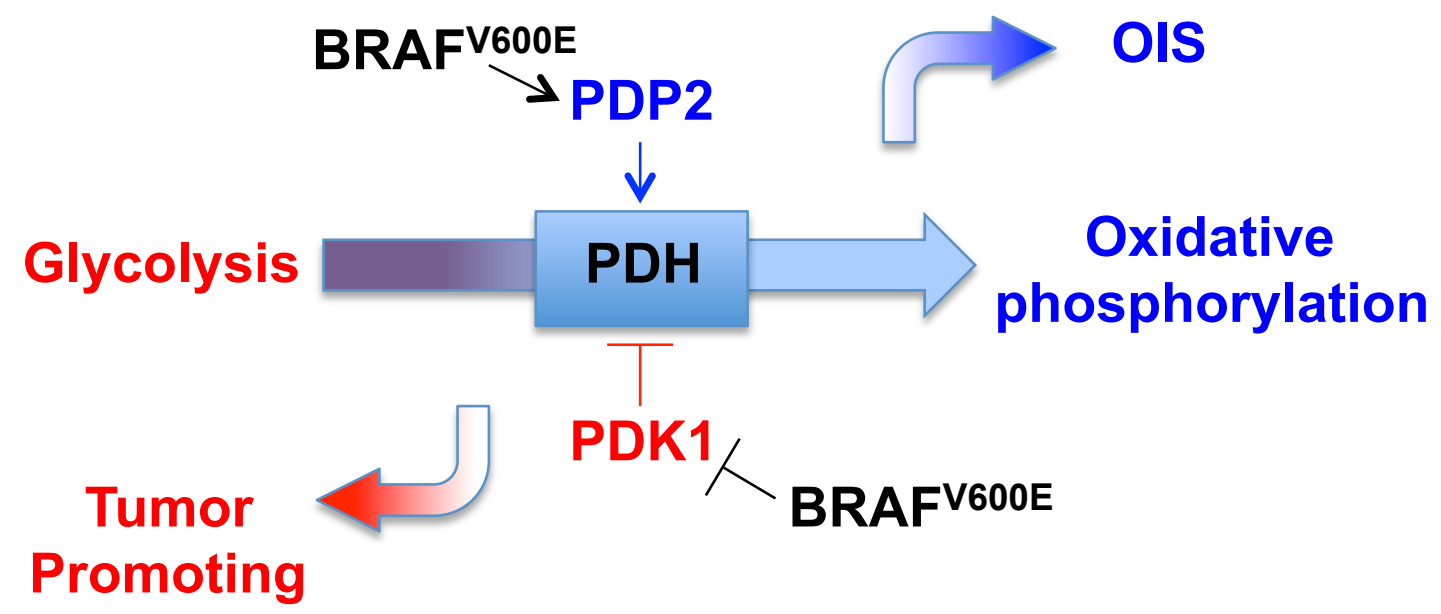

Figure 1: Model for regulation and function of the PDK1-PDP2-PDH axis in OIS and tumor progression 